\title{
Alginate Microspheres: The Innovative Approaches to Production of the Microbeads/Micro-Particles
}

\author{
K. Venkata Ramana Reddy ${ }^{1}$, Jaideep Gupta ${ }^{2}$, Pathan Izharuddin ${ }^{2}$ \\ 1. Research Scholar*, Acharya Nagarjuna University, Guntur, Andhra Pradesh, India- 522510 \\ 2. KVK College of Pharmacy, Surmaiguda, Ranga Reddy Dist, Telangana State-501512
}

\begin{abstract}
Multiparticulate dosage form is pharmaceutical formulations where the active substance is in the form of a number of small independent subunit such as, granules, microparticle, beads, and microspheres. Multiparticulate drug delivery systems are specifically suitable for achieving delayed and sustained release oral formulations with a minimum risk of dose dumping, local irritation, systemic toxicity, and variation in rate of bioavailability as they are less dependent on gastrointestinal transit time and offers many advantages over unit particulate dosage forms. Microbeads are small, sphere shaped, systematically produced free-flowing properties, semi-spherical solid units. Microbeads are the agglomerates of about size ranging from $0.5 \mathrm{~mm}$ to $1.0 \mathrm{~mm}$, obtained from various starting materials and utilizing different innovative techniques. A blend of multiparticulate can also be used to provide the desired drug release profile. These round shaped particles produc ed by mostly principle of atomization technique.Among various different innovative techniques Vibration technology, Electrostatic bead generator, Jetcutter technology, electrostatic forces, dropping method and a mechanical cutting device are few of many. In current article it describes about the total methods involved and their working principles with diagrams were clearly illustrated. Now a days sodium alginate beads were designed by these technologies mostly in relative to microencapsulation techniques.
\end{abstract}

Keywords: Atomization, Vibrating technology, Jetcutter device, Electrostatic bead generator Dropping method and electro-spray technology.

Article Info: Received 13 June 2019; $\quad$ Review Completed 12 August 2019; $\quad$ Accepted 20 August 2019; Available online 25 August 2019

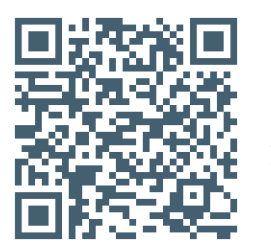

Cite this article as:

Ramana Reddy KV, Gupta J, Izharuddin P, Alginate Microspheres: The Innovative Approaches to Production of the Microbeads/Micro-Particles, Journal of Drug Delivery and Therapeutics. 2019; 9(4-s):774-781

http://dx.doi.org/10.22270/jddt.v9i4-s.3413

K. Venkata Ramana Reddy, Research scholar, Department of Pharmaceutics, Acharya Nagarjuna University, Nagarjuna Nagar, Guntur, Andhra Pradesh, India -522510

\section{Introduction}

\section{Air atomization:}

Atomization may be a strategy in which the disruptive action of externally applied aero-dynamic forces is restricted by the merging the each influences of the liquid consistency and surface tension forces. In air spray atomization, liquid rising from a fixed nozzle at low frequency is encircled by highspeed stream of air. Air atomization needs requires utilization of an air blower to possess the choice to create atomizing air that required shearing the oil into tip. In air atomization needs piping for air, solenoid valves and air pressure regulators.
Friction between the air and liquid encourages and interrupts the fluid stream and initiates the procedure of atomization. The energy supply for causing air atomization is air pressure. The operator will manage the stream rate of liquid autonomously of the energy supply. Diagram of it was showed in figure 1\&2.reflects the stream of liquid going through a little breadth of the opening; as it shows a fast stream of air encloses the liquid stream. Note that other modules will cover the performs of the horns you see on the representation and therefore shower designs ensuing spray pattern. Remember that it is the relative distinction in velocity among fluid and air that causes the atomization procedure. From the availability of different atomization systems, air-blast or twin fluid atomization has been normally used suspension splash drying 1 . 

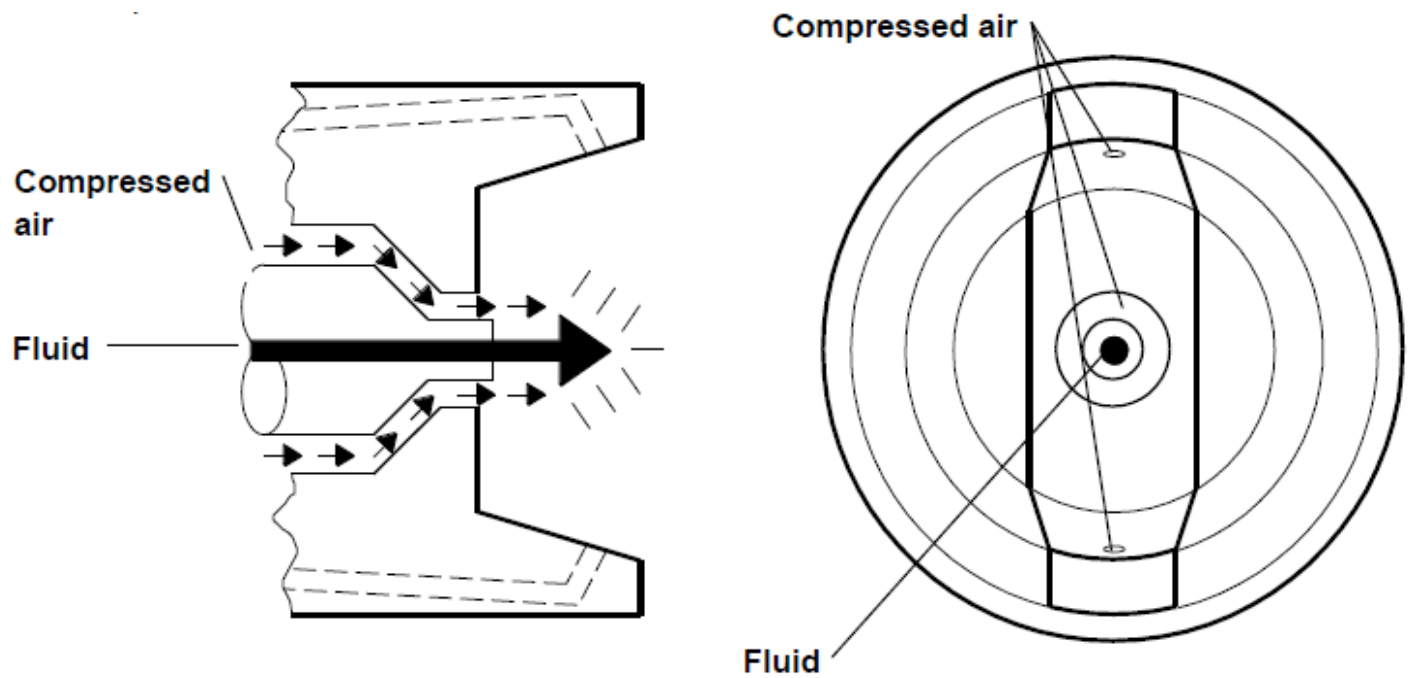

Figure 1: The relative velocities of air and fluid for airless and air spray atomization (Close view)

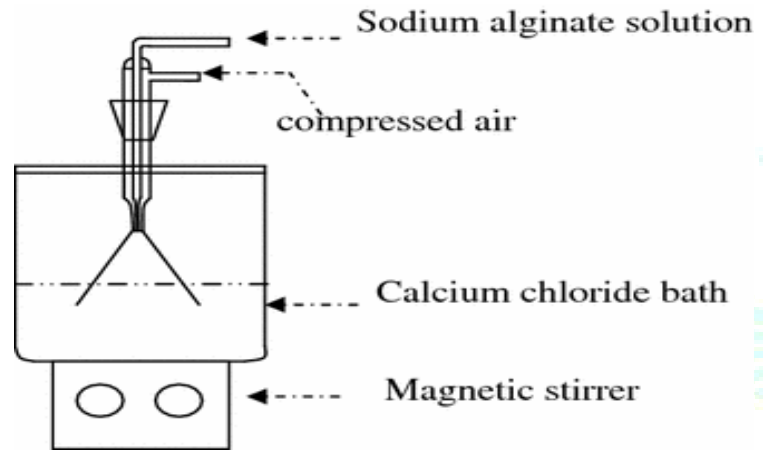

Figure 2: Diagram of air atomization technique.

\section{Spinning disc atomization:}

The liquid slag was set in an induction furnace. In the wake of softening, the temperature of the liquid slag was kept up at $1700 \mathrm{~K}$. The molten liquid slag was poured onto the spinning disk that was run by a motor at the base. The movement of slag pursued the gravity force before contact with the disk surface and afterward splashed out move toward becoming grind and small particles. The experiment was spot with utilizing a high-speed video camera. The granulated slag was gathered on a molecule gathering sheet. This picture reveals that the granulation process of the molten slag started out as a slim film flowing from the discharged slag on the center of the SDA disk during rotation. At that point, the film splashed out of the disk due to centrifugal force to form a number of various ligaments. At last, the ligaments knock off to become granulates and were cooled while falling movement, as shown in Fig.3. In this manner, a comprehensive granulation model was built by these three modes slender film, tendon, and drop development. This model initially assesses the physical and compound properties of the slag and computes the film thickness on the circle edge utilizing the scalar condition strategy trailed by ascertaining the quantity of tendons.
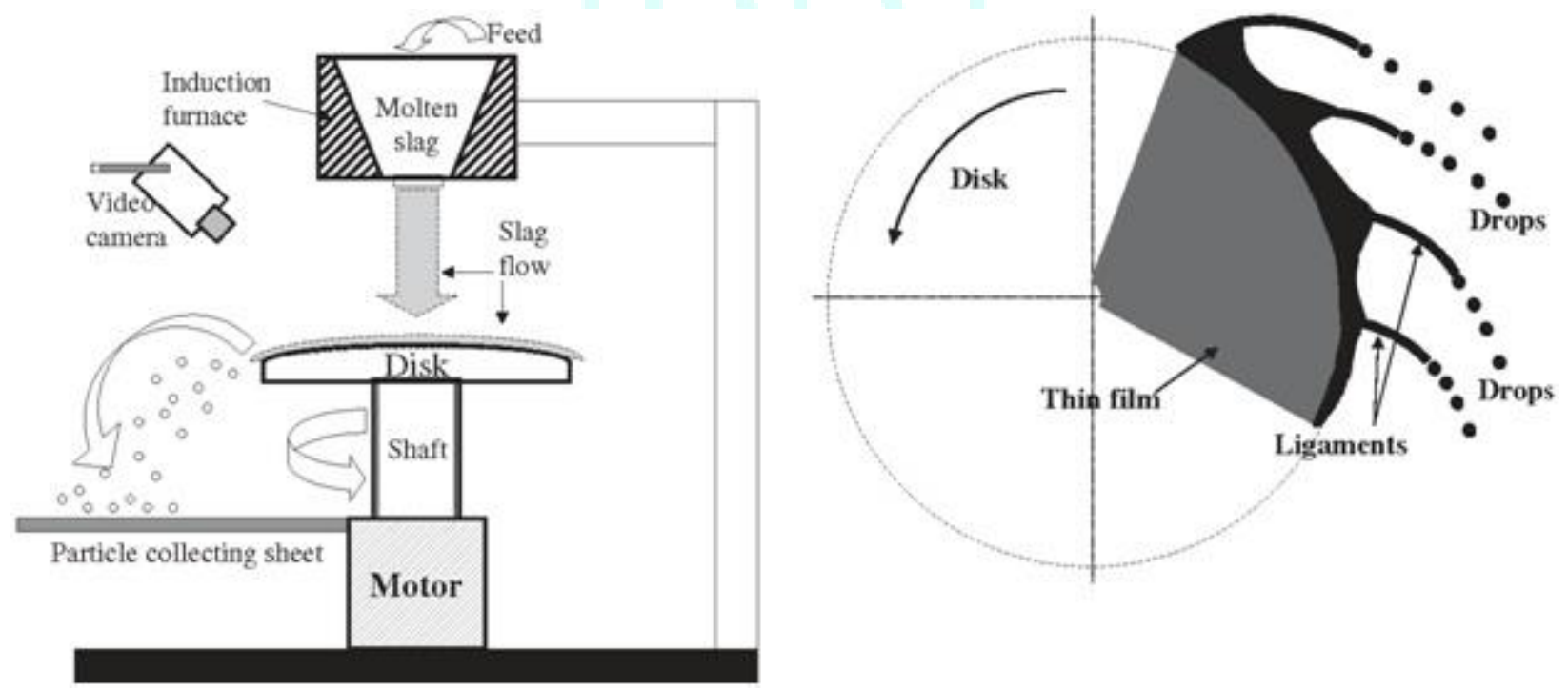

Fig 3: Diagram of Spinning Disc atomization.

In spinning disk atomization process microcapsules get ready by spinning disk atomization, within in which the fluid breakup is evoked by a rotating disk, have sizes starting from 350 to $600 \mu \mathrm{m}$. 


\section{Vibrating nozzle process:}

In this, a liquid suspension containing active compound material, that to be make encapsulated by placing in an immobilization solution, is constrained into a small opening; the subsequent liquid jet is put into a mode of vibration and in later it causes to breaks up into fine droplets that are then solidified to form small particles. In any case, the big hurdle to be finding out in this process is the consistency of the solution to be implemented. It is highly not possible to break up the jet with higher viscosities of $50 \mathrm{cps}$. This type of device forms capsules with diameters within the range of $300-600 \mathrm{~m}^{2,3}$.

\section{The Coaxial Bead Generator:}

The coaxial airflow dropping that produces microcapsules within the range $400-800 \mu m^{4-6}$. In Coaxial air or liquid flow type the coaxial air jet system is a basic method for producing small beads (down to around 350-400 $\mu \mathrm{m}$ ), in spite of the fact that the size dissemination will regularly be bigger when contrasted with an electrostatic framework. In this framework, a coaxial air stream is utilized to pull beads from a needle tip into a gelling medium.

\section{Coaxial flow for liquid and air:}

The air fly coaxial framework is a simple technique for moderately little globules generation (around $400 \mu \mathrm{m}$ ), despite the fact that the distribution size demonstrates a slight augmentation in contrast with the electro-static operating system. In this work, a coaxial stream of the air is controlled to get out the droplets from the tip of the needle to direct the gelling bath before they would fall because of gravity.

\section{Coaxial gas flow bead generator:}

The essential guideline of the instrument is the utilization of a coaxial air stream which pulls beads from a needle tip into the gelling bath. The instrument is appropriate for the generation of circular beads to reduce size of beads to around $600 \mu \mathrm{m}$. In the coaxial option, there is an extra fit arrangement for the feeding of the shell polymer solution.
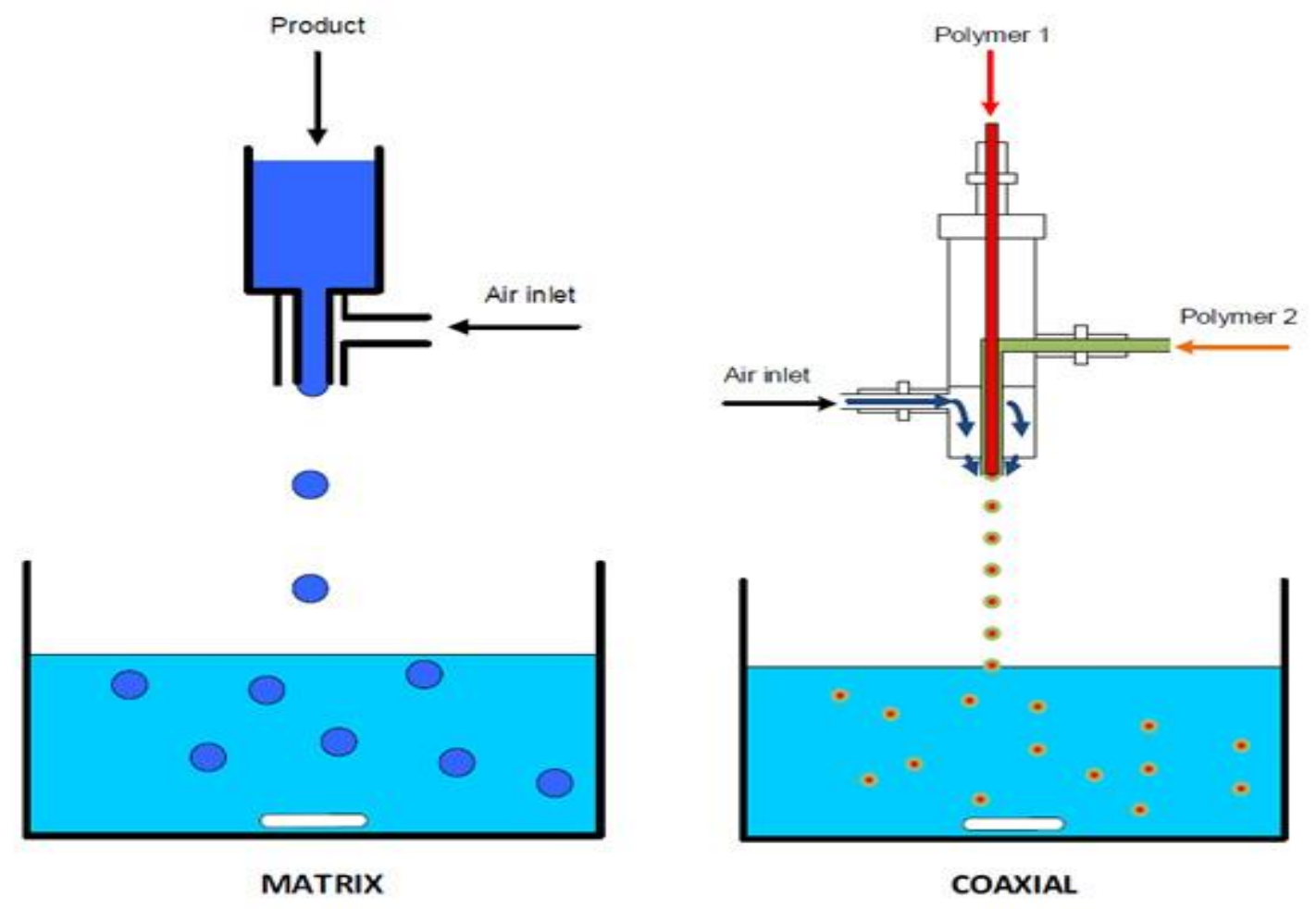

Fig 4: Schematic representation of Coaxial Flow for air and liquid.

\section{Jet Cutter technology:}

It works on a mechanical cut of a liquid jet by continuing rotating cutting wires positioned in a cutting tool. Basically based on the working of principle of rotating wires that cut falling jets of the hydrogel precursor solution into little pieces. These are cut at normal interims, framing indistinguishably measured drops that are gelled in a crosslinking shower as they fall. The Jet Cutter can be worked either in the "ordinary mode" or in the "soft landing mode". Normal mode means that the Jet Cutter put some place somewhere at a definite height and the beads are collected in a $\mathrm{CaCl}^{2}+$ bath placed on the uniform floor space. Softlanding mode means that the JetCutter is placed on the floor and the beads are collected at a height of about $2 \mathrm{~m}$, this reduces the velocity of the bead when entering the gelation bath. In soft landing mode, (Fig.5). The speed of the spherical beads is drastically decreased close to the zenith of the direction where they are gathered. Henceforth, the speed at which the beads enter the fluid gathering shower is somewhat low also; the danger of bead distortion is diminished. In the ordinary (normal) mode, the path directions of the beads are somewhat immediate from topdown. The two methods of this innovation produce capsules inside the range $330-800 \mathrm{~m}$. The formed droplet size mainly depends on rotation frequency of the cutting tool,the nozzle diameter, number and wires diameters. 


\section{normal mode}

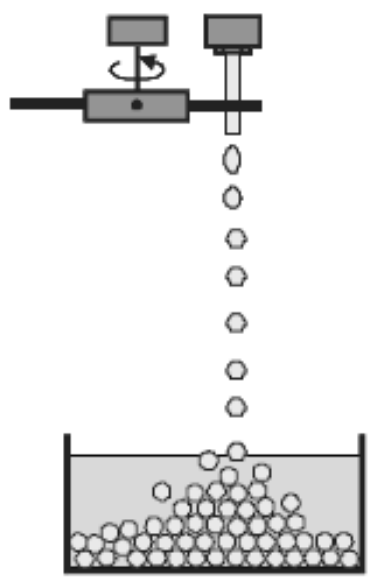

soft landing mode

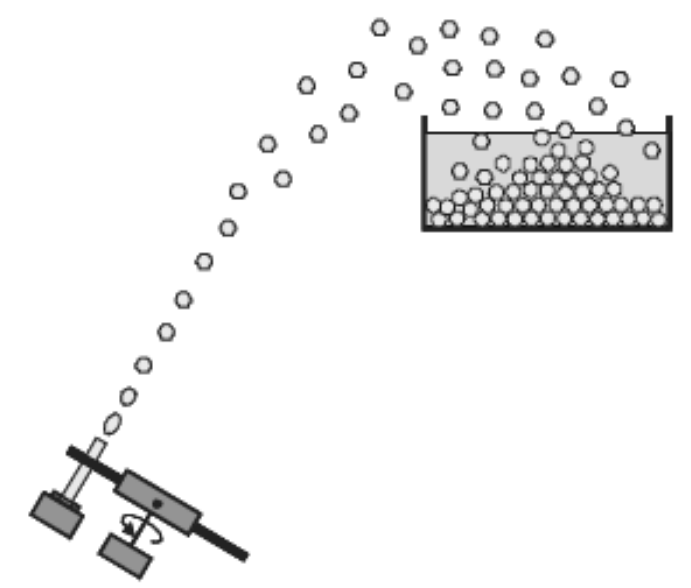

Fig 5. Scheme of the two JetCutter modes for production of beads.

\section{Electrostatic bead generator:}

The new methodology is predicated based on the implement of the electrode, especially on its type. The ring electrode is put in between the nozzles and the hardening solution. The beads fall through the centre of the electrode. Electrostatic droplet generation produces microcapsules by an electrostatic pulse generator at intervals the vary range vary 300-800 m A new technique supports based on a microfluidics device, that use a silicon micro-nozzle array, have achieved reduce the diameter of the capsules to $162 \mathrm{~m}$. Alginate is extruded through the micro-nozzle and is shared by the viscous drag force of oil flow to create calcium alginate gel beads (fig.6). The instrument utilizes an adjustable electrostatic voltage of a few $\mathrm{kV}$ between the nozzle and gelling medium so as to scale to drop diameter of the droplet size. Different nozzles are available for the selection of desired bead size in market.

\section{The following factors influence the bead size:}

- Diameter of needle.

- Gap in between the needle and electrode.

- Fixation of voltage rate.

\section{Advantage of electrostatic generator compared to the standard nozzle holder:}

1. The diameter of bead is independent from the level of the hardening/curing solution.

2. With conical jetting the bead become generally smaller than in comparison with conventional technology.

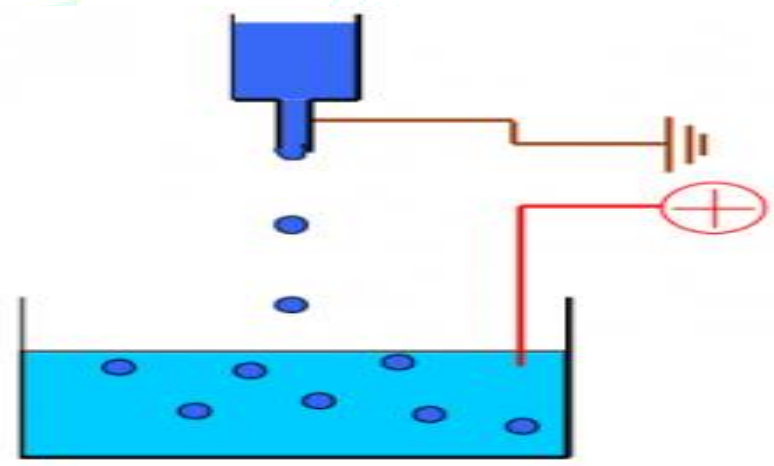

Principle of eletrostatic bead generator

Fig 6: Diagram of Electrostatic bead generator.

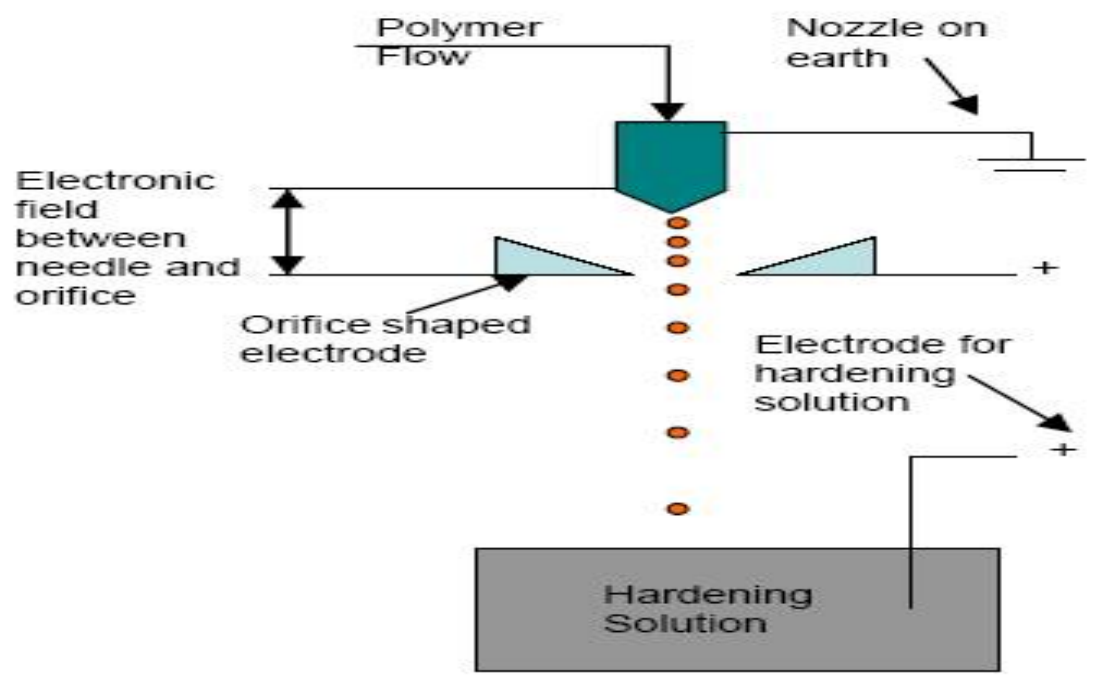

Fig 7: Brief view of Electrostatic Bead Generator. 


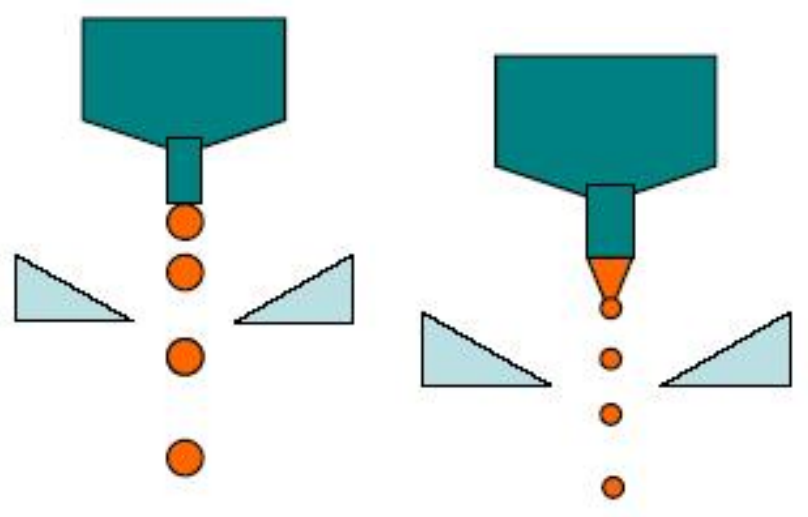

Fig 8: Low electrostatic field with standard nozzle holder (Left side), High electrostatic field with conical jetting electrostatic accelerator resulting in small beads (Right side).

\section{Coaxial air-flow}

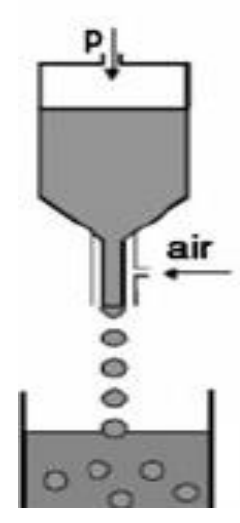

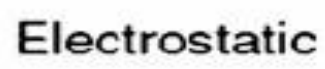

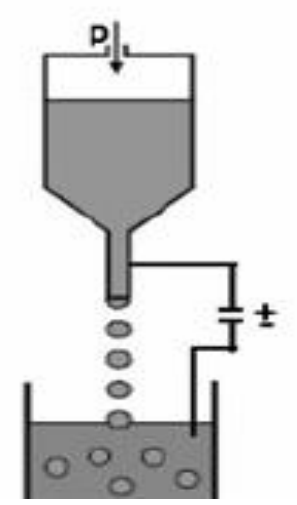

Vibration

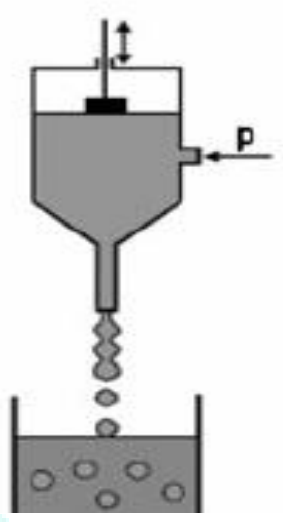

JetCutter

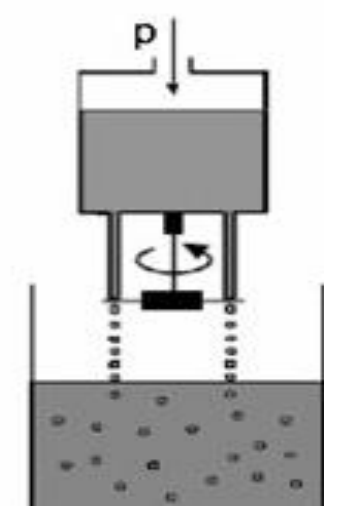

Fig 9: Comparative view of Bead Productions by Various Techniques.
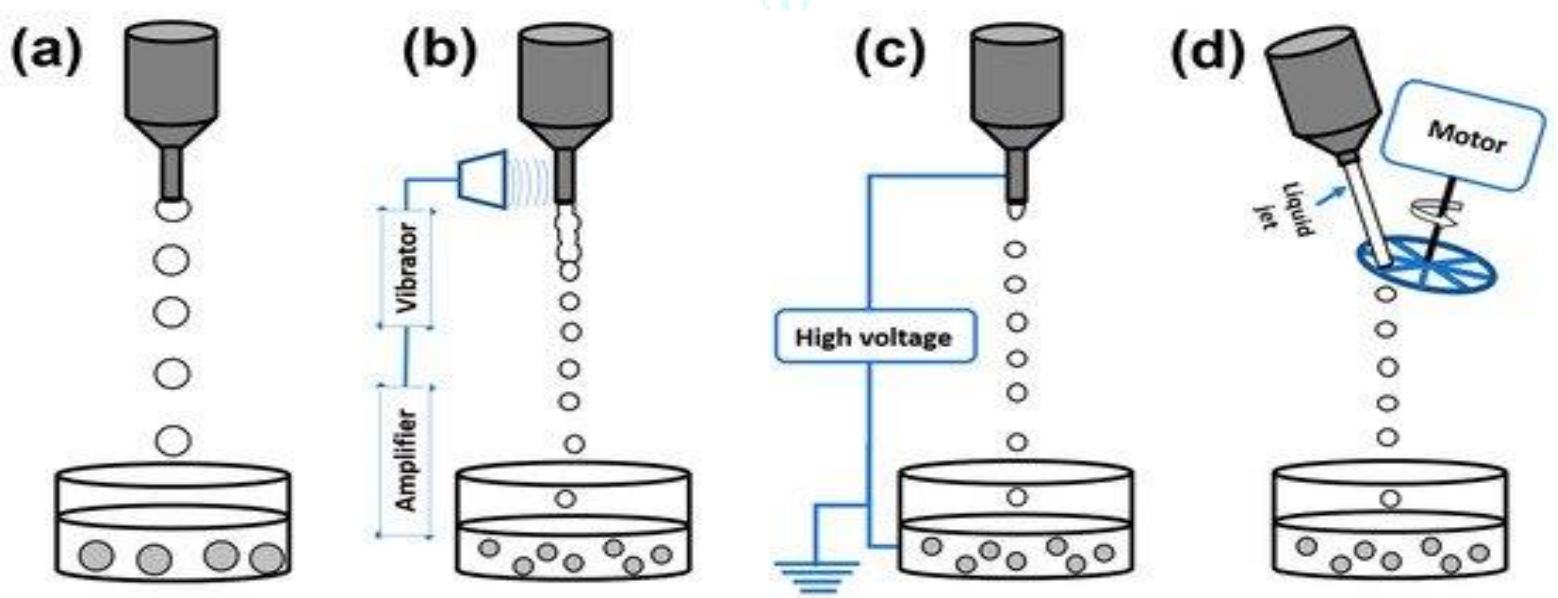

Fig 10 : Illustration of dropping devices: (a) conventional dropping method influenced by gravity, surface tension and viscosity; breaking up of liquid jets into droplets stimulated by (b) vibrating nozzle method, (c) electrostatic forces and (d) a mechanical cutting device.

Four of these advancements, the coaxial airflow, electrostatic, vibration, and JetCutter technology, were utilized for bead production in this trail. Calcium alginate hydrogel beads with the diameter of $800 \pm 100 \mu \mathrm{m}$ were delivered by the most widely recognized bead production technologies utilizing $0.5-4 \quad$ mass $\%$ sodium-alginate solutions beginning material. Dynamic consistency of the alginate solutions ranged from extended from under 50 mPas up to more than $10,000 \mathrm{mPas}$. With the coaxial air flow and electrostatic enhanced dropping advancement as well as with the Jet Cutter technology in the soft-landing mode, beads were formed from all sodium alginate solutions, whereas the vibration technology was not suitable of processing of using the high viscosity $3 \%$ and $4 \%$ sodium alginate solutions. Spherical shape beads were created by utilizing both electrostatic and the Jet Cutter technologies. Slightly deformed/abnormal shape beads were obtained from high-viscosity sodium alginate solutions by utilizing 
the coaxial air flow and from the $0.5 \%$ and $2 \%$ sodium alginate solutions using the vibration technology. The rate of bead generation utilizing the Jet Cutter was around multiple times higher than with the vibration technology and in excess of multiple times higher than with the coaxial wind current and electrostatic technology, Thus, the Jet Cutter was found to be the best innovative technology for enormous scale/modern applications despite the fact that it isn't constrained to such scales and can likewise be utilized for little/lab scale applications. The vibration technology can likewise be utilized in any scale despite the aspect that the lower throughputs compared with the Jet Cutter, require multi-nozzle devices for larger-scale applications. The coaxial air-flow and the electrostatic technologies are constrained to small/lab-scale applications ${ }^{7}$.

\section{Vibrating nozzle technique.}

In this, a liquid suspension containing active compound material, that to be make encapsulated by placing in an immobilization solution, is constrained into a small opening; the subsequent liquid jet is put into a mode of vibration and in later it causes to breaks up into fine droplets that are then solidified to form small particles. In any case, the big hurdle to be finding out in this process is the consistency of the solution to be implemented. It is highly not possible to break up the jet with higher viscosities of $50 \mathrm{cps}$. This type of device forms capsules with diameters within the range of $300-600 \mathrm{~m}^{8-10}$. A vibrating nozzle generates drops from a pressurized vessel. In this experiment, protein-immobilized based calcium alginate beads created with uniform shape littler than $20 \mu \mathrm{m}$ in size by utilizing a vibration technology. Calcium alginate beads production solution containing isopropyl alcohol (IPA), isoamyl alcohol and aqueous $\mathrm{CaCl}^{2+}$ in proportion of a $2: 1: 1$ was added into a $1.5 \mathrm{ml}$ test tube.
Sodium alginate polymer solution (alginate concentration: 1 $\% \mathrm{~g} / \mathrm{ml}$ FITC-labeled BSA) $\mu \mathrm{w} / \mathrm{w}$ ) containing protein (25 l GL $\mu$ l syringe by a syringe pump through a fused silica capillary $\mathrm{m}$ ) (GL Sciences) at a constant flow rate (0.1$2 \mu(30-75 \mathrm{l} / \mathrm{min})$ and dropped into the physical mixture slowly, while $\mu$, Visaton, $\Omega$ vibrating with a loudspeaker which was associated to a sine wave sound generator to produce cross-linked calcium alginate beads. The frequency of the sine wave sound generator was fixed exactly to 200 Hz. To harvest the calcium alginate beads produced, the test tube was centrifuged at frequency of 5,000 rpm for 3 minutes. The upper iso amyl alcohol phase was rejected, taking consideration not to remove the soft calcium alginate beads. After adding $100 \mathrm{mM} \mathrm{CaCl}{ }^{2+}$, the whole suspension was blended using a micro-tube mixer until the precipitated calcium alginate beads were completely re-suspended. Centrifugation was run at a speed of $5,000 \mathrm{rpm}$ for 5 minutes. This washing step was repeated at least 2-3 times cautiously, and the last volume was changed in accordance with $50 \mathrm{ml}$. The bead size is adjusted mainly by the diameter of nozzle and the wavelength although also other parameters e.g. influence the droplet formation and viscosity.

\section{Electro-spraying technique:}

Electro-spraying technology encourages the generation of small and practically uniform beads and these beads having higher diffusion rate bringing about procedure execution improvement. There are few key variables influencing both particle size and shape behavior of electro sprayed sodium alginate beads, in meantime collaboration these components present multifaceted nature in deciding suitable conditions to deliver round beads with the size of interest.

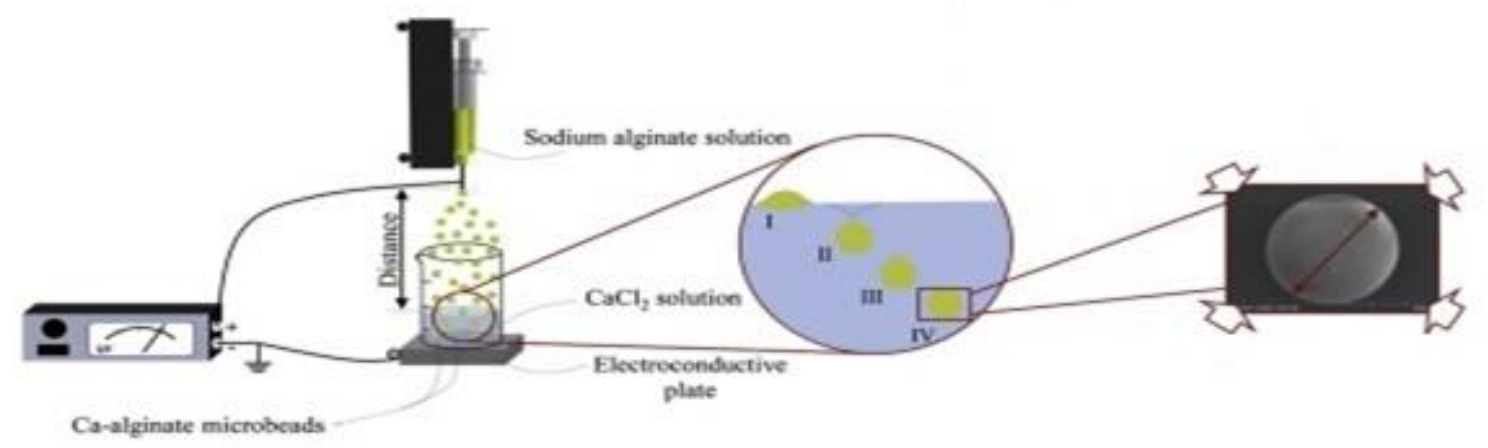

Fig 11: Diagram of Electro Spraying Technique.

\section{Electrostatic Potential Bead Generator:}

The electro-static potential is considered to be useful in pulling out the droplets from the base of the needle into the gelling bath. An electronic potential is applied between the needle feeding the sodium alginate solution and an electroconductive solution underneath. The major effect on the development of the droplet through the electrostatic potential can legitimately upgrade the charged atoms to the superficial layer of the droplet to overcome the tension of the surface. The voltage forces the beads to tumble off the needle tip before it has developed to the point where it tumbles off because of its own weight. The beads are shaped when droplets fall into the solution. Inside the utilization of this sort of instrument, the beads size lower than $200 \mu \mathrm{m}$ and with a small size distribution might undergo generation. The focus on bead size is accomplished effectively by the instrument voltage adjustment (electro-static potential). The real line of smaller beads fabrication by using electro-static potential bead generators is illustrated in following figure. 

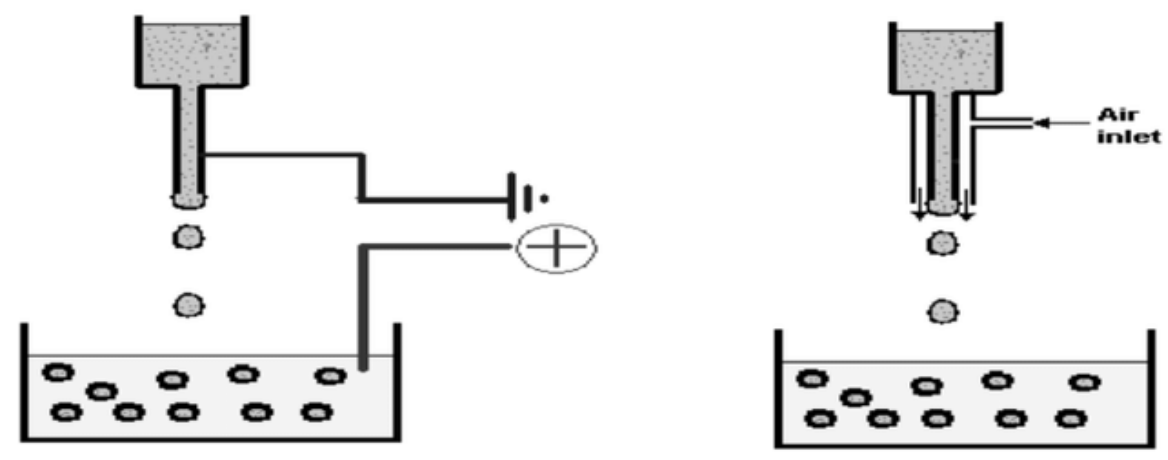

Fig 12: Diagram showing working principle of Electrostatic Bead Potential Generator.

\section{Rotating capillary jet breakage:}

The generation of the bead is procured by means via the removal a rigid jet of fluid bulging of a nozzle by utilization of cutting device having the rotational limit. The fluid is then segmented into cylindrical parts then forming the beads because of surface tension ability when passing the gelling bath. Ex: JetCutter. JetCutting Hydrogel spherical shaped particles were formed by using the Jet Cutter. The removal of the polymer solution through the nozzle was constrained by compressed air from the house supply line. A schematic illustration of the jet cutting process is displayed in Figure 13. The throughput was adjusted with fixation of a pressureregulating valve. Sprinklers with various estimation of 250-
$1000 \mu \mathrm{m}$ were used to change particle size. A cutting, liquid cylinder fell downwards into the collection bath. All Through falling, cylinders formed spherical droplets due to the principle of surface tension of the polymer solution. A reasonable collection bath was placed at a distance of around 50 or $80 \mathrm{~cm}$ beneath the cutting tool. The volume of the gelation bath was at least five times the total volume of the processed polymer solution to enable good stirring and particle separation. Subsequent to completing the jet cutting, the content of the collection baths was stirred with a suitable magnetic bar for at least ten more minutes to guarantee complete gelation of formed particles and to keep away from agglomeration. Gelled particles were expelled from gelation shower by means of separating process.

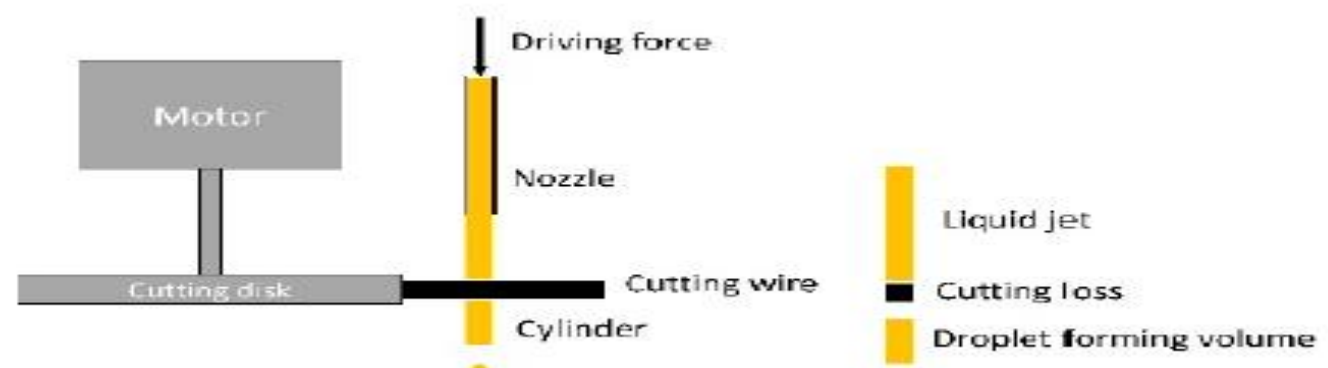

Droplet

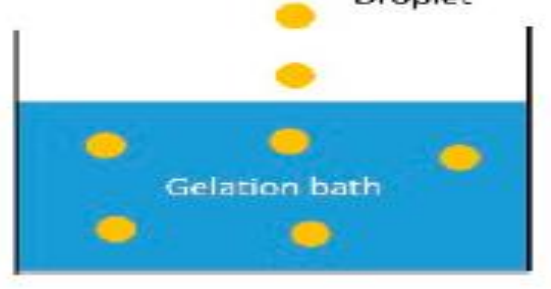

Fig 13: Schematic View of Rotating Capillary Jet Breakage.

\section{Emulsification process ${ }^{11}$ :}

is a process wherein the mixing of two non-immiscible liquids is stabilized by an emulsifying agent which is often act as a surfactant. In this method, liquid may be formed into small spherical droplets in an immiscible liquid medium. Continuous energy input such as blending or agitation and adding suitable surfactants which keep the dispersed phase in the continuous phase as it is a dynamic process. Once a stable emulsion is prepared (for instance, water-in-oil, w/o), physical and chemical impact may generate gelation and further it makes to cause stabilization of the droplets water- to-oil proportion (w/o) is one of the key parameters influencing emulsification process. Or the ratio most part runs from $1: 2$ to $1: 10$ at the lab scale. A change from lab to bulk scale production clearly some conversation starters like the ideal proportion as well as the oil recovery. Generally, the ratio of the viscosities of the aqueous to the oil phase needs to be below one in order to break the fine droplets. The surfactant concentration and its hydrophilic-lipophilic balance (HLB) worth out to likewise be considered to make stabilization of the emulsion droplets. With every single other condition kept constant, an increase of the blending 
rate from 200 to $500 \mathrm{rpm}$ prompt to reduce in the particle size by a factor of 5.1 for a turbine with baffles, though just by a factor of 2.7 for marine impeller.

\section{Laminar jet break up Technique:}

The electromagnetic laminar jet breakup technology is based on the principle that a laminar jet of a liquid feed passing a nozzle of appropriate diameter is broken into equally sized droplets by a super-imposed mechanical vibration. The vibration has to be done in resonance of the PlateauRayleigh instability and leads to very uniform/equal droplets. Vertically falling stream of water will break up into drops if its length is greater than about 3.12 to 3.17 times its diameter. Using the natural liquid instability and replacing the natural irregular disturbances with the regular permanent mechanical vibration, small uniform droplets are formed. The droplets are further processed in order to form particles by polymerization or cross-linking or other hardening/ curing process of the liquid sustained. The particle diameter can be postulates and managed by selecting the factors like nozzle diameter, superimposed frequency and volumetric flow

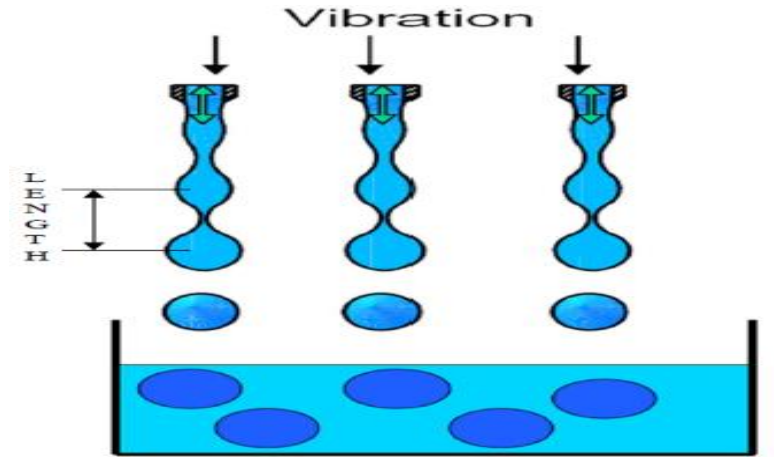

Fig 14: Diagram of bead production by Vibrating Technology.

\section{Dropping method:}

The principle involved in dropping method is natural gravity. The liquid goes through the tip of the nozzle; drops grow and separate from the stream, at that point fall into the hardening/curing solution. Constant permanent flow is maintained. Gravity drop generation is the simplest method of drop production of all available techniques. The distance between medium and tip of nozzle will decide diameter of soft beads.

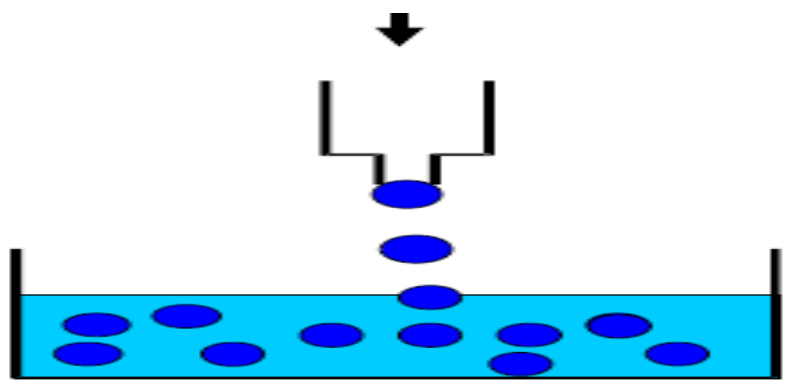

Fig 15: Diagram of Bead Production by Dropping Method.

\section{Complex Coacervation process:}

Coacervation might be started in various ways. Examples are changing the temperature, $\mathrm{pH}$ or including a second substance for example concentrated aqueous ionic salt solution or a non-solvent. As the coacervate forms, it must moist the suspended core particles or core droplets and coalesce into a continuous coating for the process of microencapsulation to happen. The last step for microencapsulation is the hardening of the coacervate wall and the separation of the microcapsules; for the most part it is the most troublesome advance in the all-out procedure. The acidic or basic nature of the system is influenced to produce microcapsules. Over a certain critical $\mathrm{pH}$ value, the system relying upon its acidic or basic nature may create microcapsules. Below that $\mathrm{pH}$ value, they won't be shaped. Normally, complex coacervation deals with the system containing more than one colloid complex coacervation can be induced in systems having two dispersed hydrophilic colloids of inverse electric charges. Balance of the general positive charges on one of the colloids by the negative charge on the accustomed to realize partition of the polymer-rich complex coacervate stage.

\section{Conclusion:}

As all above described methods offers many advantages than current technology and along with procedure of working, these methods were influenced by specified parameters, design and its working principle. The getting chances of accessibility, reliability, precision and accuracy were more in these innovative approaches.

\section{References}

1. Sugiura S, Oda T, Izumida $Y$, Aoyagi $Y$, Satake M, Ochiai A, Ohkohchi N, Nakajima M., Size control of calcium alginate beads containing living cells using micro-nozzle array. Biomaterials. 2005; 26:3327-3331.

2. Serp D, Cantana E, Heinzen C, Von Stockar U, Marison I.W., Characterization of an encapsulation device for the production of monodisperse alginate beads for cell immobilization. Biotechnol. Bioeng. 2000; 70:41-53.

3. Shwinger C, Klemenz A, Busse K, Kressler J., Encapsulation of living cells with polymeric systems. Macromol. Symp. 2004; 210:493-499.

4. Shwinger C, Klemenz A, Busse K, Kressler J., Encapsulation of living cells with polymeric systems. Macromol. Symp. 2004; 210: 493- 499.

5. Canaple L, Rehor A, Hunkeler D., Improving cell encapsulation through size control. J. Biomater. Sci. Polym. 2002; 13:783796.

6. Robitaille R, Pariseau J.F, Leblond F.A, Lamoureux M, Lepage Y, Halle J.P., Studies on small l $\left(<350^{\prime} \mathrm{m}\right)$ alginate-poly-L-lysine microcap sules. III. Biocompatibility of smaller versus standard microcapsules. J. Biomed. Mater. Res. 1999; 44:116120.

7. Maria Raytchinova Kosseva., Immobilization of Microbial Cells in Food Fermentation Processes. Food Bioprocess Technol. 2011; 4:1089-1118.

8. Serp D, Cantana E, Heinzen C, Von Stockar U, Marison I., Characterization of an encapsulation device for the production of monodisperse alginate beads for cell immobilization. Biotechnol. Bioeng. 2000; 70: 41-53

9. Shwinger C, Klemenz A, Busse K, Kressler J., Encapsulation of living cells with polymeric systems. Macromol. Symp. 2004; 210: 493-499.

10. Yan Zhou, Shinichiro Kajiyama, Hiroshi Masuhara, Yoichiro Hosokawa, Takahiro Kaji, Kiichi Fukui., A new size and shape controlling method for producing calcium alginate beads with immobilized proteins. J. Biomedical Science and Engineering, 2009; 2:287-293.

11. Boyd J, Parkinson C, Sherman P., Factors affecting emulsion stability, and the HLB concept. J. Colloid Interface Sci. 1972; 4:359-370. 